\title{
Evaluation of Sporadic and Familial Cases with Craniofrontonasal Syndrome: A Wide Clinical Spectrum and Identification of a Novel EFNB1 Gene Mutation
}

\author{
Semra Gürsoy ${ }^{\mathrm{a}}$ Filiz Hazan ${ }^{b}$ Tülay Öztürk ${ }^{c}$ Rüya Çolak ${ }^{d}$ Şebnem Çalkavur $^{d}$

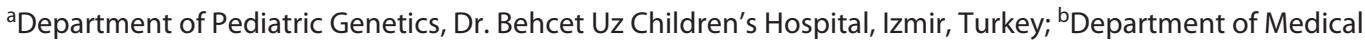 \\ Genetics, Dr. Behcet Uz Children's Hospital, Izmir, Turkey; 'Department of Pediatric Radiology, Dr. Behcet Uz \\ Children's Hospital, Izmir, Turkey; ${ }^{d}$ Department of Neonatology, Dr. Behcet Uz Children's Hospital, Izmir, Turkey
}

\author{
Keywords \\ Craniofrontonasal syndrome · Craniosynostosis · \\ Dysmorphic facial features $\cdot$ EFNB1 - Central polydactyly
}

polydactyly and deep palmar creases may be part of the clinical spectrum seen in CFNS. In addition, our findings expand the mutational spectrum in patients with CFNS.

(C) 2021 S. Karger AG, Basel

\section{Introduction}

Craniofrontonasal syndrome (CFNS; OMIM 304110) is a rare $\mathrm{X}$-linked dominant disorder which was first described by Cohen [1979]. The main characteristic features of CFNS include severe, often asymmetric hypertelorism, a short and wide upper face, a central nasal groove, craniofacial asymmetry, uni- or bicoronal craniosynostosis, sloping shoulders, ridging and longitudinal splitting of the nails, mild cutaneous syndactyly, duplication of the first digit, and wiry curly hair. Additionally, corpus callosum agenesis or dysgenesis, cleft lip and palate, umbilical and diaphragmatic hernia have been noted [Fischer et al., 2016; Acosta-Fernández et al., 2020]. Interestingly, CFNS presents paradoxically with heterozygous females more severely affected than hemizygous males. Hemizygous males also show no or only a mild manifestation such as hypertelorism [van den Elzen et al., 2014]. The difficulties establishing boundaries in the mosaic state karger@karger.com

www.karger.com/msy
(C) 2021 S. Karger AG, Basel
Correspondence to:

Semra Gürsoy, dr.semra@hotmail.com

\section{Karger"}


arising from $\mathrm{X}$ inactivation intermingling EFNB1-negative and EFNB1-positive cells (cellular interference), aberrant functional disomy of the $\mathrm{X}$ chromosome, and a functional homologue on the $\mathrm{Y}$ chromosome that possibly ameliorates the effect of the $\mathrm{X}$ chromosome mutation might explain the variable expression of the disease [Twigg et al., 2004; Davy et al., 2006]. Impaired signaling capacity of ephrin-B1 and dysregulation of gap junctional communication could also be responsible for the expression of the disease [van den Elzen et al., 2014].

Loss-of-function mutations in the EFNB1 (ephrin B1) gene, which encodes ephrin-B1 protein, are responsible for this syndrome. EFNB1 is located in the long arm of the $\mathrm{X}$ chromosome (Xq13.1) and comprises 5 exons [Wieland et al., 2004]. Ephrin-B1 is involved in the formation of the boundry of neural crest and mesoderm; therefore, CFNS has been termed as a neurocristopathy. It has been suggested that CFNS phenotypes may be attributable to impacts on early neural crest cell migration or later bone differentiation defects [Niethamer et al., 2020]. Signaling between EPH receptors and EPHRINs is also important for cell migration, repulsion, and adhesion during neuronal, vascular, epithelial development and maintains normal cell to cell interactions via proper establishing tight junctions. The protein comprises different domains. The extracellular ephrin domain is encoded by exons 2 and 3; the transmembrane and intracellular domains are encoded by exon 5 . The protein also has an $\mathrm{N}$-terminal signal peptide which is essential for protein secretion and is then subsequently cleaved from the mature protein [Darling and Lamb, 2019; Nakai, 2000].

More than a hundred EFNB1 mutations have been linked to CFNS. In $94 \%$ of these pathogenic variants, missense, nonsense, frameshift, and splice site mutations have been identified. The majority of these mutations are located in exons 2 and 3. Partial or complete gene deletions were detected in the remaining patients (6\%); contiguous gene deletions, including EFNB1, were also revealed in a few cases [Wieacker and Horvath, 2016; Romanelli Tavares et al., 2019]. In the present study, we aimed to evaluate the clinical characteristics and molecular findings of 2 sporadic and 2 familial cases with CFNS.

\section{Materials and Methods}

\section{Study Patients}

Four female patients with CFNS from 3 unrelated Turkish families referred to our Pediatric Genetic and Medical Genetic Departments due to their dysmorphic features were included in the study. A detailed clinical and genetic evaluation was performed by a clin- ical geneticist. A clinical diagnosis of CFNS was made on the basis of dysmorphological features of the patients. Demographic, clinical, laboratory findings, direct radiographic and $3 \mathrm{D}$ cranial CT data were collected from the medical records of the patients. Due to lack of specific growth charts for CFNS, the growth parameters of the patients were measured and evaluated according to the growth charts established for Turkish infants and children. Denver developmental screening test (under 6 years of age) and Wechsler Intelligence Scale for Children-Revised test (6-16 years of age) were used to investigate developmental delay/intellectual disability in the pediatric individuals.

\section{DNA Extraction and Sanger Sequencing}

Genomic DNA was extracted from the peripheral blood lymphocytes of all patients and their parents with QIAamp DNA Blood Mini Kit (Qiagen GMBH, Hilden, Germany) using standard procedures. Thereafter, we amplified the EFNB1 gene by polymerase chain reaction (PCR) and analyzed all of the coding exons and exon-intron boundries of the gene by Sanger sequencing. The sequences were analyzed using CLC Genomics Workbench 3 sequencing program (Qiagen). The Ensembl database (GRCh38.p13) with ENST00000204961.5 transcript ID of EFNB1 was used to compare the individuals and the reference sequence. All variants were searched in ClinVar (https://www.ncbi.nlm.nih.gov/clinvar/), Leiden Open Variation Database (LOVD: www.lovd.nl/) and $\mathrm{Hu}-$ man Gene Mutation Database (HGMD, Institute of Medical Genetics, Cardiff, http://www.hgmd.org/) as reference databases. We also used the search engine Varsome (https://varsome.com/), which has information from 30 external databases, to investigate the pathogenicity of the novel variant. The pathogenicity of the identified sequence variants was reported using an automatic variant classifier that evaluated the submitted variant according to the American College of Medical Genetics (ACMG) guidelines, classifying it as one of "pathogenic", "likely pathogenic", "likely benign", "benign" or "uncertain significance" [Richards et al., 2015].

\section{Results}

\section{Clinical Reports}

The detailed clinical features and molecular findings of 4 female patients from 3 different families are presented in Table 1.

Familial Patients (patients 1 and 2)

A 4.5-year-old girl (patient 1) was consulted for evaluation of dysmorphic features. She was born at full term gestation with a low birth weight $(2,000 \mathrm{~g})$. There was no consanguinity between the parents, and facial features of the mother (patient 2) were similar to her daughter (Fig. 1a-b). Patient 1 had severe hypertelorism, upslanting palpebral fissures, facial asymmetry, strabismus, midline nasal groove, short nose, posteriorly rotated ears, and wiry hair. Examination of the extremities revealed proximally placed thumb, deep palmar creases, overlapping toes, and 


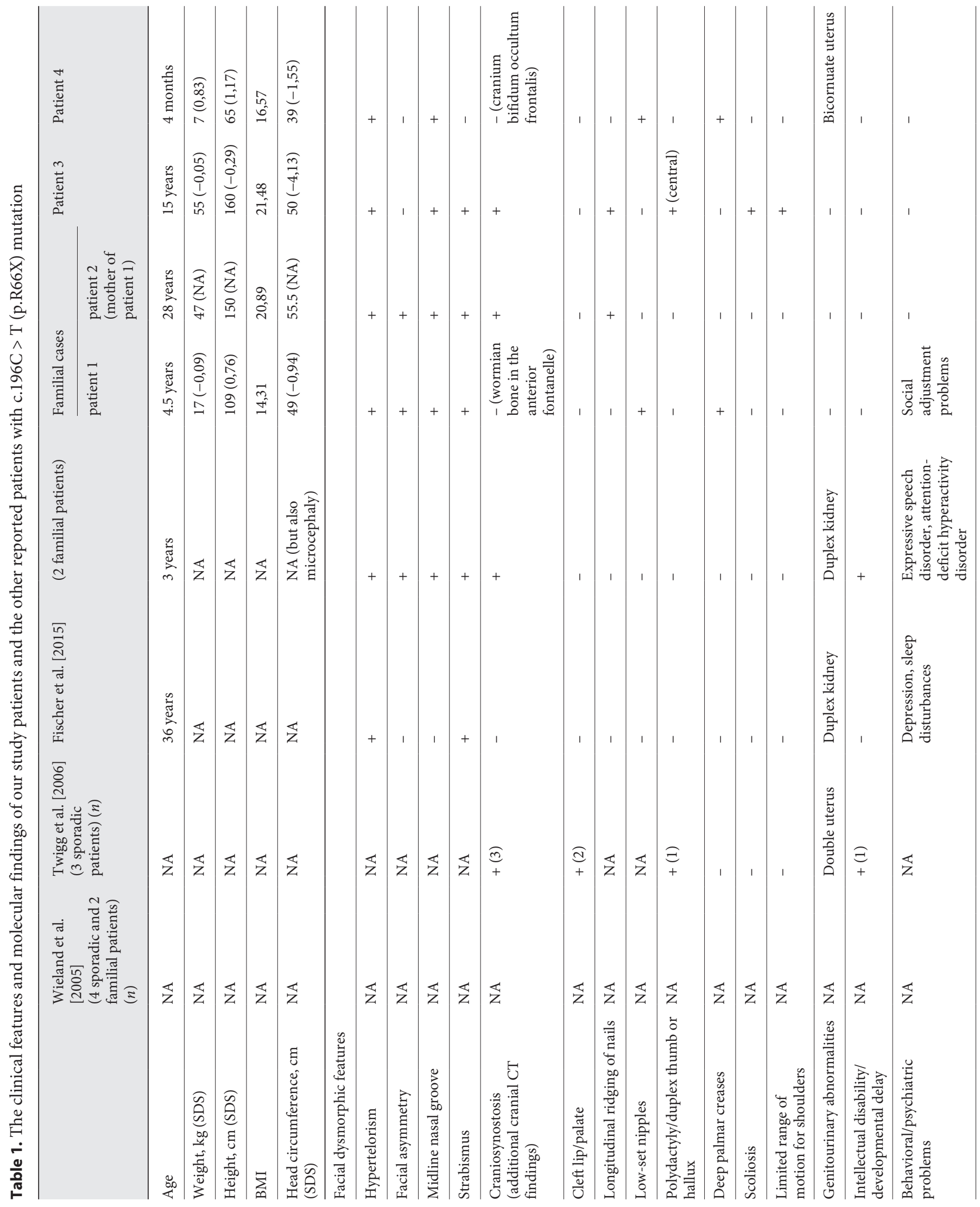




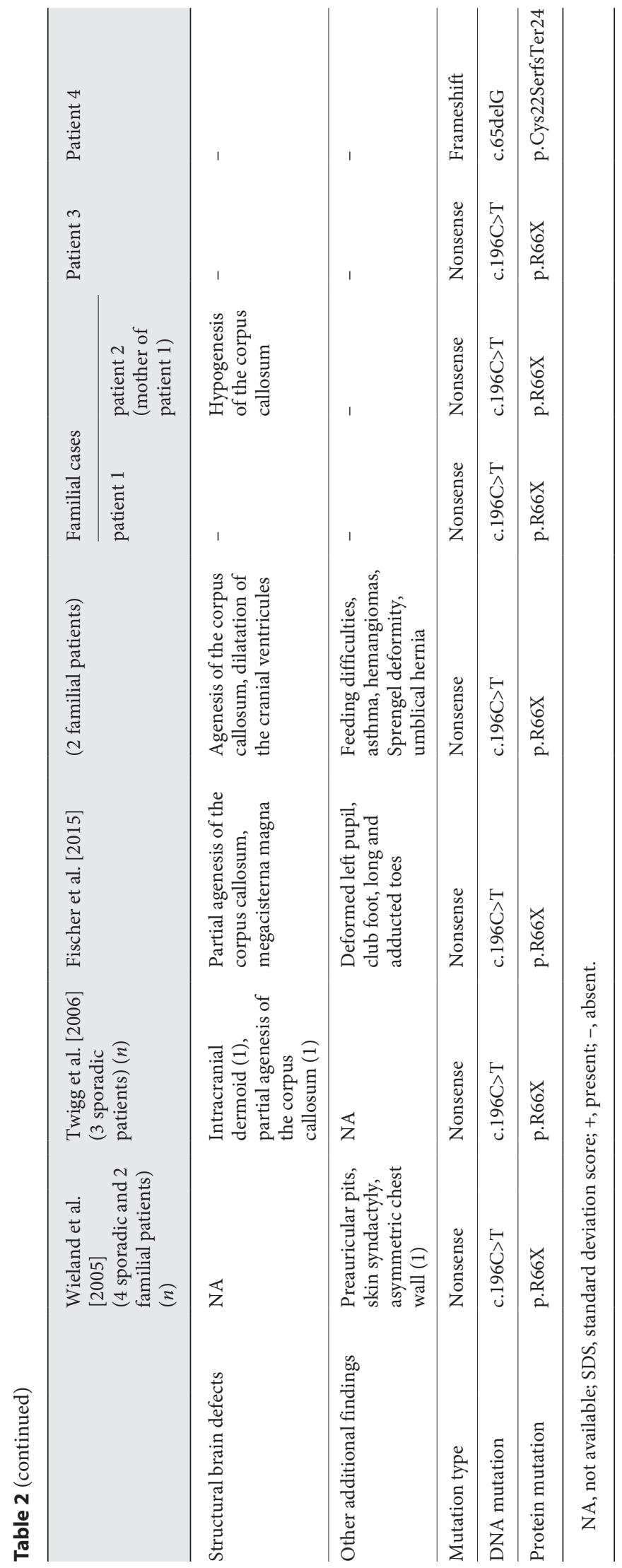




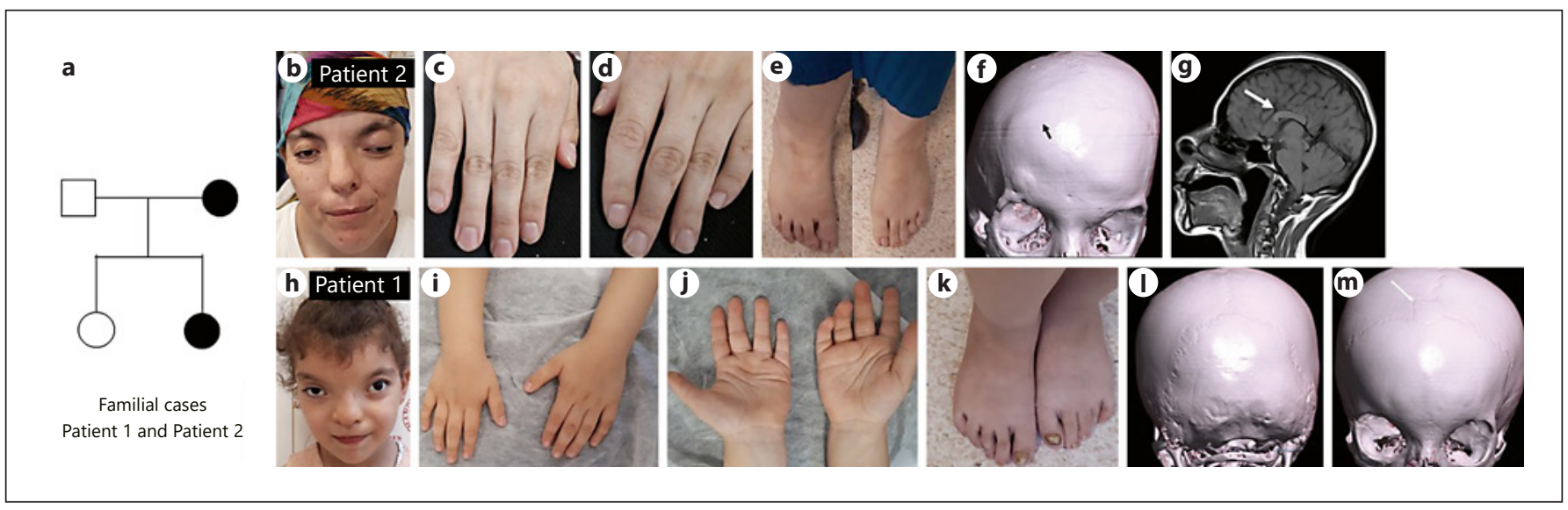

Fig. 1. The detailed clinical and radiological findings of the familial patients (patients 1 and 2). a The pedigree of the familial cases. b-e Clinical features of patient 2 including unilateral proptosis and unilateral ptosis, facial asymmetry, midline nasal groove, longitudinal splitting of nails, clinodactyly (2nd, 4 th and 5 th fingers) of the left hand, a widely spaced gap between the first and second toes, and brachydactyly of the fourth and fifth toes. f CT scan results showing unilateral coronal craniosynostosis (arrow). g Cra- nial MRI demostrates hypogenesis of the corpus callosum (arrow). h-k Dysmorphic features of patient 1 including hypertelorism, upslanting palpebral fissures, facial asymmetry, strabismus, midline nasal groove, wiry hair, proximally placed thumb, deep palmar creases, overlapping toes, and fungal infection of the first toenails. I, $\mathbf{m}$ CT scan results showing normal cranial sutures and wormian bone in the anterior fontanelle (arrow).
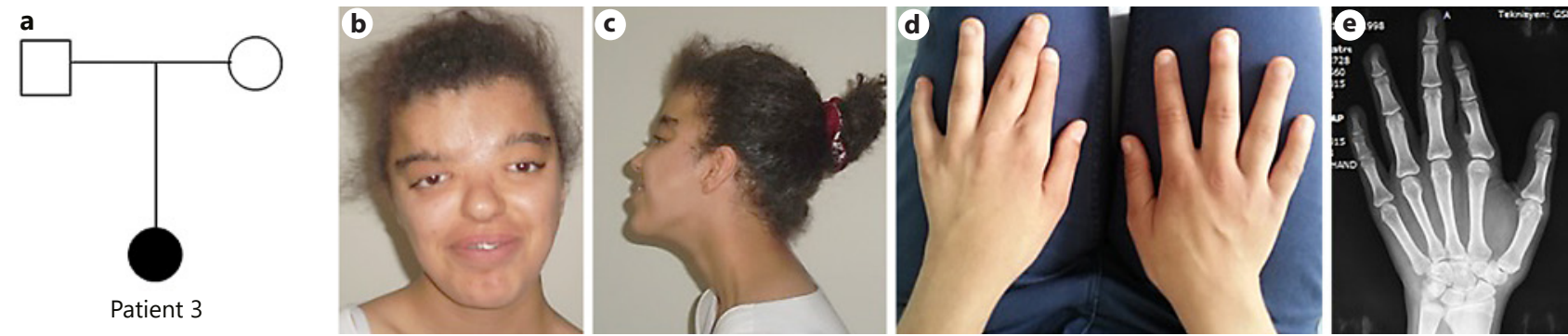

Fig. 2. The clinical and radiological findings of patient 3. a Pedigree of the patient. b-d Clinical features of patient 3 showing hypertelorism, downslanting palpebral fissures, aberrant form of eyebrows, broad nasal root, midline nasal groove, and a surgical scar between the second and third digits of the left hand. e Direct radiography of the left hand after operation.

fungal infection of the first toenails (Fig. 1h-k). Additionally, a hyperpigmented macule on the right arm and lowset nipples were detected. Her weight was $17 \mathrm{~kg}(-0.09$ SDS), her height was $109 \mathrm{~cm}$ (0.76 SDS), and head circumference was $49 \mathrm{~cm}$ (-0.94 SDS). She had normal developmental skills, but had social adjustment problems at the beginning of preschool education. Abdominal ultrasonography (USG), echocardiography, and hearing test results were normal. All cranial sutures were open and wormian bone in the anterior fontanelle was detected in the 3D cranial CT (Fig. 1l, m). The mother (patient 2) had additional clinical findings including unilateral coronal

Clinical Variability of Patients with

Craniofrontonasal Syndrome craniosynostosis, unilateral proptosis and unilateral ptosis, longitudinal splitting of nails, clinodactyly (second, fourth, and fifth fingers) of the left hand, a widely spaced gap between the first and the second toes, and brachydactyly of the fourth and fifth toes (Fig. 1b-f). Brain MRI revealed hypogenesis of the corpus callosum (Fig. 1g).

\section{Sporadic Patients}

\section{Patient 3}

A 15-year-old female patient was referred to our department due to dysmorphic facial features. She was the only child of a healthy couple. She was born at term after 

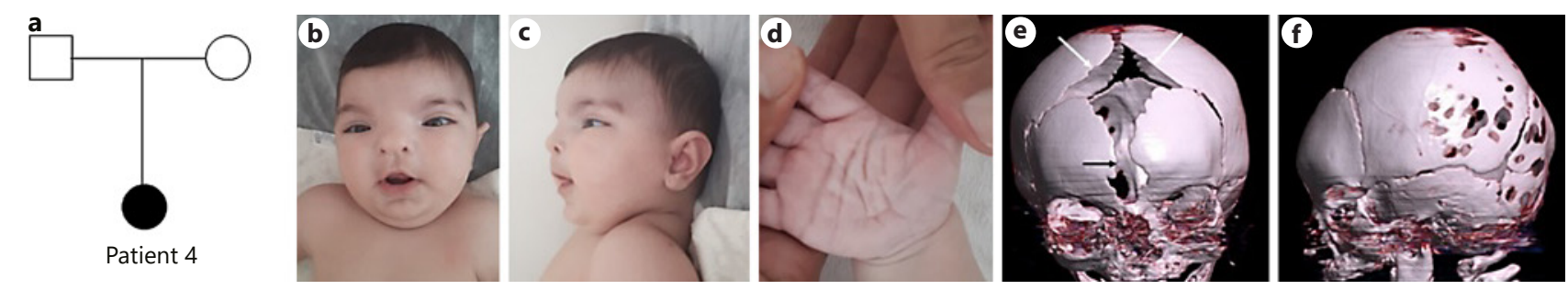

Fig. 3. The clinical findings and CT scan results of patient 4. a Pedigree of the patient. b-d Dysmorphic features of patient 4 including hypertelorism, depressed nasal bridge and nasal tip, broad nasal bridge, midline nasal groove, downturned corners of mouth, midface retrusion, thin upper lip, triangular philtrum, and short neck. e, f $3 \mathrm{D}$ cranial CT demostrating cranium bifidum occultum frontalis and abnormally large anterior fontanelle (arrows).

an uneventful pregnancy. Her birth weight was 3,600 g (0.7 SDS), and her birth length was $50 \mathrm{~cm}(0.28 \mathrm{SDS})$. Unilateral (right) coronal craniosynostosis and central (meso-axial) polydactyly (between the $2 \mathrm{nd}$ and $3 \mathrm{rd}$ digits of the right hand) were detected; she was operated in the early childhood due to these abnormalities. On admission, her height was $160 \mathrm{~cm}$ (-0.29 SDS), her weight was $55 \mathrm{~kg}(-0.05$ SDS $)$ and head circumference was $50 \mathrm{~cm}$ (-4.13 SDS). During physical examination, hypertelorism, strabismus, downslanting palpebral fissures, aberrant form of eyebrows, broad nasal root, midline nasal groove, high palate, webbed neck, sloping shoulders, limited range of motion for shoulders, and longitudinal splitting of nails were detected (Fig. 2a-c). A surgical scar was noted between the second and third digits of the left hand (Fig. 2d, e). The patient had normal intellectual development. Abdominal USG, cranial MRI, and echocardiographic studies were also unremarkable. Ophthalmologic examination did not reveal any abnormality. Hearing test results were normal. Spinal radiographs revealed thoracolumbar scoliosis. A physiotherapy was scheduled for the scoliosis and limited range of motion in the shoulders.

\section{Patient 4}

A 6-day-old female newborn was evaluated in our genetic clinic for dysmorphic facial features. She was born to healthy parents by cesarean section after 40 weeks of uncomplicated gestation. Her birth weight was 3,800 g (1.17 SDS), birth height was $50 \mathrm{~cm}(0.28$ SDS) and birth head circumference was $35 \mathrm{~cm}$ (0.36 SDS). Clinical examination showed hypertelorism, depressed nasal bridge and nasal tip, broad nasal bridge, underdeveloped ala nasi, midline nasal groove, downturned corners of mouth, midface retrusion, thin upper lip, and a triangular phil- trum. Additionally, brachycephaly, short neck, inverted and widely spaced nipples, low-set nipples, a hyperpigmented macula in abdominal area, deep palmar creases, pes planus, and overlapping toes were detected (Fig. 3ad). At 4 months of age, she had a weight of $7 \mathrm{~kg}(0.83$ SDS), height of $65 \mathrm{~cm}$ (1.17 SDS) and a head circumference of $39 \mathrm{~cm}(-1.55$ SDS). Eye examination and hearing tests were normal. Skeletal survey (skull, entire spine, ribs, pelvis, long bones radiographs) did not reveal any significant radiological findings. Cranial CT showed that all cranial sutures were open. Additionally cranium bifidum occultum frontalis and abnormally large anterior fontanelle were revealed on 3D cranial CT (Fig. 3e-f). Transfontanel USG and echocardiography were also normal. Bicornuate uterus was detected on abdominal USG. Her developmental milestones were found to be compatible with her age.

\section{Molecular Results}

The heterozygous nonsense mutation in the EFNB1 gene (c.196C>T; p.R66X), which was previously reported in 3 different studies [Wieland et al., 2005; Twigg et al., 2006; Fischer et al., 2016], was detected in the familial cases (patients 1 and 2) and also in a sporadic case (patient 3) in our study. Wieland et al. [2005] reported 4 sporadic and 2 familial cases with this nonsense EFNB1 gene mutation and clinical evaluation was described in only 1 sporadic patient [Wieland et al., 2005]. Additionally, 3 sporadic female patients with the same mutation, c.196C>T; p.R66X, were reported by Twigg et al. [2006]. The patients had variable clinical features, but all of them had unilateral or bilateral craniosynostosis, and 2 of them had cleft lip/palate [Twigg et al., 2006]. A mother and a 3-year-old daughter were also reported by Fischer et al. [2016]. The clinical findings 


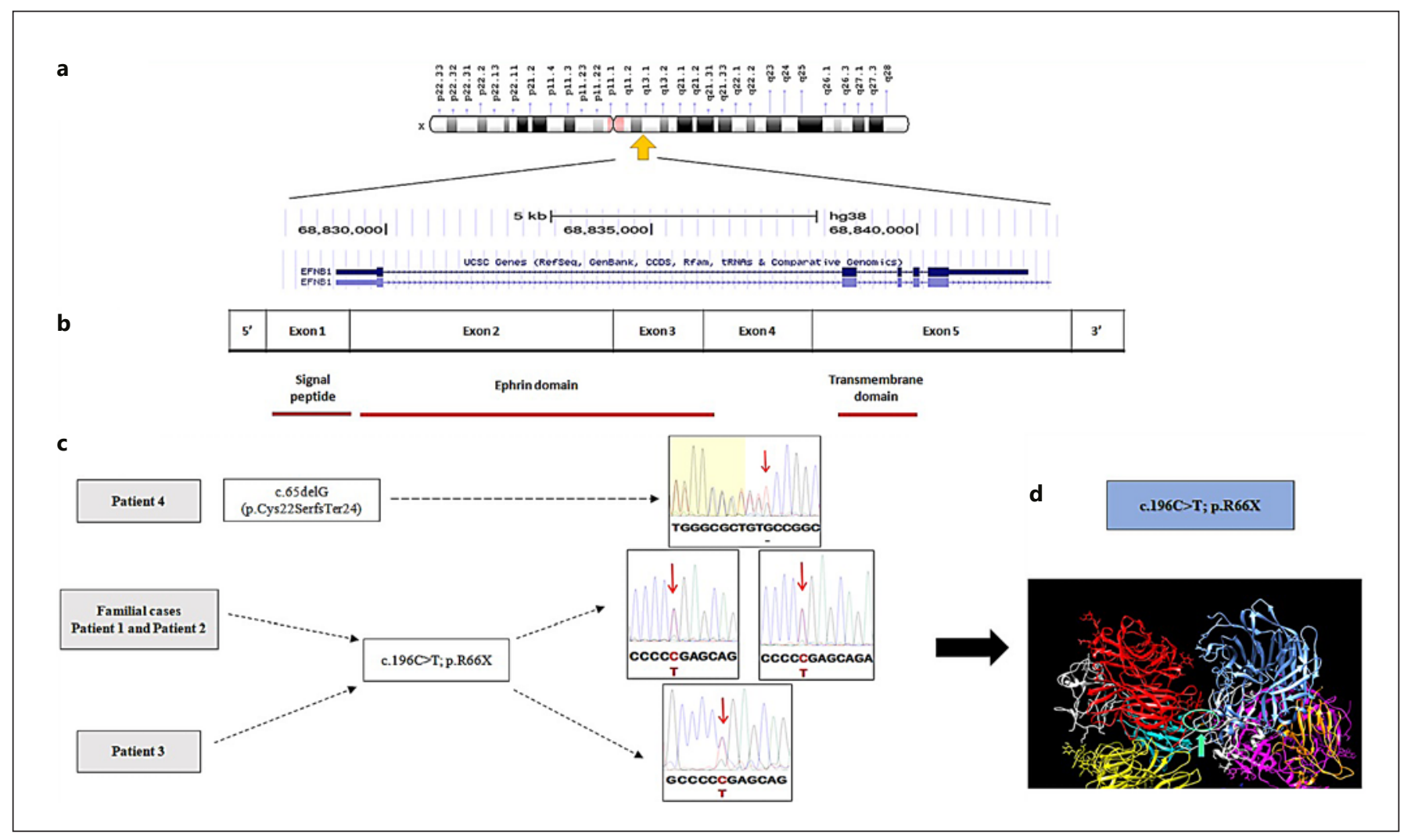

Fig. 4. Schematic illustration of the EFNB1gene, structure of the protein, and Sanger sequencing results of the patients. a The scheme describes the chromosomal location of EFNB1 using UCSC Genome Browser (GRCh38/hg38). b The exon structure of EFNB1 and the encoded protein domains. c Sanger sequencing results of the patients. $\mathbf{d} 3 \mathrm{D}$ model of the wild-type ephrin $\mathrm{B} 1$ pro-

of these reports [Wieland et al., 2005; Twigg et al., 2006; Fischer et al., 2016] were compared with the current study and are listed in Table 1 . This mutation was located in exon 2 and caused a premature stop codon. On the other hand, mutational analysis of both parents of patient 3 was normal.

Additionally, in patient 4, Sanger sequencing analysis revealed a heterozygous nucleotide deletion, c.65delG; p.Cys22SerfsTer24, in exon 1 of EFNB1 and N-terminal signal peptide of the protein, which caused a frameshift variation that is likely to lead to loss of function in the protein. This mutation has not been previously reported in the literature, but according to ACMG guidelines, it is predicted as a pathogenic variant (PVS1, PM2, PP3). Molecular findings of the parents showed a wild-type sequence. The detailed results of these 2 mutations are presented in Figure 4.

Clinical Variability of Patients with

Craniofrontonasal Syndrome tein. The green circle shows the location of the nonsense mutation c.196C>T; p.R6×6 (using the website https://www.uniprot.org/ uniprot/P98172). Protein model of ephrin B1 does not contain the $\mathrm{N}$-terminal signal peptide domain, so we were not be able to demonstrate the structure of the frameshift mutation, c.65delG; p.Cys22SerfsTer24.

\section{Discussion}

In this study, we presented the characteristics of $4 \mathrm{fe}-$ male patients from 3 different Turkish families with CFNS. A central polydactyly and deep palmar creases, which have not been previously described, are reported for the first time in this syndrome. A novel EFNB1 gene mutation has also been identified.

The molecular spectrum of EFNB1 includes missense, frameshift, nonsense, and splice site mutations, with a predominance of frameshift and nonsense mutations resulting in premature truncation codons [Wieland et al., 2005]. To date, 104 different variants have been reported in the Leiden Open Variation Database. Pathogenic and likely pathogenic mutations have accounted for one-fifth of these mutations (18 pathogenic, 3 likely pathogenic) [Fokkema et al., 2011]. The majority of mutations are located in exons 2 and 3, coding for the ephrin domain of the pro- 
tein. In our report, 2 different mutations were detected. One of them was, c.196C>T; p.R66X, a nonsense mutation which was located in exon 2 and in the ephrin-domain of the protein. This mutation has been described before in both familial and sporadic cases [Wieland et al., 2005; Wallis et al., 2008; Fischer et al., 2016]. Wieland et al. [2005] reported 4 sporadic and 2 familial (mother and daughter) patients with c.196C >T; p.R66X mutations, who presented with dysmorphic craniofacial features, but detailed clinical information was not given [Wieland et al., 2005]. In another study, Twigg et al. [2006] reported 3 different sporadic patients with the same EFNB1 mutation (c.196C $>\mathrm{T}$; p.R66X). Although all of these 3 patients had craniosynostosis, a cleft lip/palate, duplex hallux and double uterus were observed in different patients. Partial corpus callosum agenesis and developmental delay were detected in only 1 patient [Twigg et al., 2006]. Additionally, several psychiatric disorders including depression and hyperactivity were described by Fischer et al. [2016] in familial patients with the same mutation. Although isolated corpus callosum agenesis itself is frequently accompanied by psychiatric symptoms, the authors suggested that dysregulated ephrin B1 signaling might be responsible for these psychiatric disorders [Fischer et al., 2016]. In our study, 3 of our patients ( 2 familial, 1 sporadic) had this mutation, and the clinical features were highly variable between these inter- and intrafamilial cases. While craniosynostosis was not detected in patient 1 , patient 2 (the mother of patient 1) and patient 3 had unilateral coronal craniosynostosis. Patient 2 also had hypogenesis of the corpus callosum; however, none of them had any behavioral or pschyiatric problems except patient 1 , who had social adjustment problems at the beginning of preschool education.

The other mutation, c.65delG; p.Cys22SerfsTer24, was a novel frameshift mutation which was detected in patient 4 and located in exon 1 of EFNB1. The mutation was also located in the $\mathrm{N}$-terminal signal peptide in which only a few mutations were reported [Wieland et al., 2005]. Twigg et al. [2004] reported 2 EFNB1 mutations which were located in exon 1 . One of the patients, who had unilateral craniosynostosis, hypertelorism, hypermetropia, astigmatism, shoulder girdle abnormalities and frizzy hair, had a nonsense mutation, c.57G $>A ; p . W 19 X$. The other patient with a missense mutation, c.1A>G; p.M1V, also had similar findings besides bilateral craniosynostosis and ridged nails [Twigg et al., 2004]. In 2006, they reported 5 additional mutations in exon 1 (c.4_4del, c.30C >T, c.109T>G, c. $110 \mathrm{G}>\mathrm{A}$, and c. $123 \mathrm{C}>\mathrm{G}$ ) in 6 patients. The patients had variable clinical features including Sprengel deformity, hypertrophic cardiomyopathy, duplex kidney, develop- mental delay and corpus callosum abnormalities. While none of them had cleft lip/palate, coronal craniosynostosis was revealed in 4 of these patients [Twigg et al., 2006]. In our study, patient 4 had only bicornuate uterus and cranium bifidum occultum frontalis in addition to her dysmorphic manifestations. Both of the mutations detected in our study were considered to be associated with loss of function of the protein. CFNS varies in phenotypic severity related to an unusual pattern of X-linked inheritance. Additionally, the random $\mathrm{X}$ inactivation could also explain the phenotypic variability in these female patients due to the interfaces between the patches with regular cell sorting and patches with wrong cell sorting. It seems that there is no clear genotype-phenotype correlation in patients with CFNS, but recently, it has been suggested that mutations in the cytoplasmic domain (exon 5) may result in lesser extracranial abnormalities compared to mutations in the ephrin domain [Han et al., 2020]. However, we did not find an obvious association between types or locations of mutations and the severity of the disease.

The phenotypic variability is very high in CFNS and different limb abnormalities including asymmetry of lower limbs, axillary pterygium, polydactyly, duplex thumb/hallux, cutaneous syndactyly, and joint laxity have been reported in the literature [Wieacker and Horvath, 2016]. In our study, central polydactyly was observed only in 1 patient (patient 3 ). It is known that preaxial and postaxial polydactyly is a feature of CFNS, but central polydactyly, which is a rarely observed duplication of any of the central 3 digits (second, third and fourth) [Yousaf et al., 2020], has not been previously reported in this syndrome. Central polydactyly is a feature found in different syndromes including Orofaciodigital syndrome, Bardet Biedl syndrome, McKusick Kaufmann syndrome, and Synpolydactyly syndromes [Schaefer et al., 2013]. The presence of another novel clinical finding, deep palmar creases, which could serve as a landmark for several different genetic disorders (e.g., Costello syndrome, Cardiofaciocutaneus syndrome, Bohring Opitz syndrome), is noteworthy in 2 patients (patients 1 and 4). CFNS affects multiple structures derived from neural crest cells. Cranial and trunk neural crest cells give rise to numerous cell types in different organs (e.g., pigment cells and Schwann cells, skeletal system, and connective tissues). Ephrin-B1 contributes to bone modeling by promoting growth plate formation and mineral formation and indirectly by inhibiting osteoclast function. Furthermore, ephrin-Eph interactions are unique due to the bidirectional signaling, and this nature of ephrin-Eph signaling is the basis of its role in coupling bone resorption 
to bone formation. Mosaic loss of ephrin-B1 also plays a role in later stages of digit formation, possibly involving perichondrium formation or maintenance [Davy et al., 2004]. These 2 novel dysmorphic features expanded the phenotypic spectrum of this syndrome, and we suggest that these rare findings might be useful during assessment of patients with suspected CFNS.

EFNB1 mutations cause a broad spectrum of cranial and body asymmetry as well as midline defects. Corpus callosum hypoplasia/agenesis is a midline abnormality of the brain and can result from disruption of numerous developmental steps from early midline telencephalic patterning to neuronal specification and guidance of commissural axons. It could be seen in approximately $30 \%$ of the patients with CFNS. The Eph/ephrin system plays a crucial role in corpus callosum development, and ephrinBs expressed on commissural axons act as guidance receptors and are known to be involved in formation of the corpus callosum and anterior and posterior commissures. [Edwards et al., 2014; Wieacker and Horvath, 2016]. In the present study, hypogenesis of the corpus callosum was detected only in patient 2 , who also had unilateral coronal craniosynostosis. Additionally, extracranial midline defects such as internal genitalial abnormalities, including duplication of the uterus, bicornuate uterus, uterus arcuatus, uterus didelphys, and septate uterus, have been previously reported in females with CFNS. These abnormalities are usually detected in patients with mensturation problems and may be associated with an increased incidence of miscarriages [Acosta-Fernández et al., 2020]. In the present report, patient 4 also had bicornuate uterus which arises when there is partial nonfusion of the müllerian ducts at the level of the fundus [Narang et al., 2019]. Bicornuate uterus has been previously reported in 2 patients with CFNS. While one of them had a deletion type mutation, a splice site mutation $($ c. $406+2 \mathrm{~T}>\mathrm{G})$ in exon 2 was detected in the other patient. None of them had any additional genitourinary abnormalities [Twigg et al., 2006; Wieland et al., 2007]. Bicornuate uterus was observed during routine diagnostic evaluation in patient 4 . Therefore, a detailed systematic assessment is crucial for detection of these midline defects and any other abnormalities.

\section{Conclusion}

In conclusion, no clear genotype-phenotype correlation is present in patients with CFNS due to high clinical variability between the individuals. However, the present

findings of the study have added novel clinical and molecular insights to the spectrum of EFNB1 mutations. Future research is required to investigate the molecular mechanisms, genotype-phenotype correlations and the possible contribution of other genetic modifiers in the expression of the EFNB1 gene in patients with CFNS.

\section{Statement of Ethics}

Samples from the patients were obtained in accordance with the Helsinki Declarations. Written informed consent for genetic testing, publication of other medical information, and photographs was obtained from all patients and/or their parents/guardians.

\section{Conflict of Interest Statement}

All authors declare that they have no conflict of interest.

\section{Funding Sources}

There was no specific funding for this study.

\section{Author Contributions}

Project design: S.G., F.H., and T.Ö.; Data collection and clinical evaluation: S.G., R.Ç., and Ş.Ç.; Sequence analysis: S.G., and F.H.; Preparation of the manuscript: S.G., F.H., T.Ö., R.Ç., and Ş.Ç.

References

Mol Syndromol 2021;12:269-278

DOI: $10.1159 / 000515697$
Acosta-Fernández E, Zenteno JC, Chacón-Camacho OF, Padilla CP, Bobadilla-Morales L, Corona-Rivera A, et al. Extracranial midline defects in a patient with craniofrontonasal syndrome with a novel EFNB1 mutation. Am J Med Genet A. 2020;182(5):1223-9.

Cohen MM Jr. Craniofrontonasal dysplasia. Birth Defects Orig Artic Ser. 1979;15(5B):85-9.

Darling TK, Lamb TJ. Emerging Roles for Eph Receptors and Ephrin Ligands in Immunity. Front Immunol. 2019;10:1473.

Davy A, Aubin J, Soriano P. Ephrin-B1 forward and reverse signaling are required during mouse development. Genes Dev. 2004 Mar 1; 18(5):572-83.

Davy A, Bush JO, Soriano P. Inhibition of gap junction communication at ectopic Eph/ephrin boundaries underlies craniofrontonasal syndrome. PLoS Biol. 2006;4(10):e315.

Edwards TJ, Sherr EH, Barkovich AJ, Richards LJ. Clinical, genetic and imaging findings identify new causes for corpus callosum development syndromes. Brain. 2014;137(Pt 6): 1579-613. 
Fischer M, Bänsch PS, Unterecker S, Romanos M, Deckert J. Depression and hyperactivity in two patients with craniofrontonasal syndrome. Am J Med Genet A. 2016 Mar;170(3): 799-800.

Fokkema IF, Taschner PE, Schaafsma GC, Celli J, Laros JF, den Dunnen JT. LOVD v.2.0: the next generation in gene variant databases. Hum Mutat. 2011;32(5):557-63.

Han JY, Kim HJ, Jang JH, Lee IG, Park J. TrioBased Whole-Exome Sequencing Identifies a de novo EFNB1 Mutation as a Genetic Cause in Female Infant With Brain Anomaly and Developmental Delay. Front Pediatr. 2020;8: 461.

Nakai K. Protein sorting signals and prediction of subcellular localization. Adv Protein Chem. 2000;54:277-344.

Narang K, Cope ZS, Teixeira JM. Developmental Genetics of the Female Reproductive Tract. In: Leung PCK, Qiao J, editors. Human Reproductive and Prenatal Genetics. 2019. p. 138-47.

Niethamer TK, Teng T, Franco M, Du YX, Percival CJ, Bush JO. Aberrant cell segregation in the craniofacial primordium and the emergence of facial dysmorphology in craniofrontonasal syndrome. PLoS Genet. 2020;16(2): e1008300.

Richards S, Aziz N, Bale S, Bick D, Das S, GastierFoster J, et al. ACMG Laboratory Quality Assurance Committee. Standards and guidelines for the interpretation of sequence vari- ants: a joint consensus recommendation of the American College of Medical Genetics and Genomics and the Association for Molecular Pathology. Genet Med. 2015;17(5): 405-24.

Romanelli Tavares VL, Kague E, Musso CM, Alegria TGP, Freitas RS, Bertola DR, et al. Craniofrontonasal Syndrome Caused by Introduction of a Novel uATG in the 5'UTR of EFNB1. Mol Syndromol. 2019;10(1-2):40-7.

Schaefer E, Lauer J, Durand M, Pelletier V, Obringer $\mathrm{C}$, Claussmann $\mathrm{A}$, et al. Mesoaxial polydactyly is a major feature in Bardet-Biedl syndrome patients with LZTFL1 (BBS17) mutations. Clin Genet. 2013;85(5):476-81.

Twigg SR, Kan R, Babbs C, Bochukova EG, Robertson SP, Wall SA, et al. Mutations of ephrinB1 (EFNB1), a marker of tissue boundary formation, cause craniofrontonasal syndrome. Proc Natl Acad Sci USA. 2004;101(23):86527.

Twigg SR, Matsumoto K, Kidd AM, Goriely A, Taylor IB, Fisher RB, et al. The origin of EFNB1 mutations in craniofrontonasal syndrome: frequent somatic mosaicism and explanation of the paucity of carrier males. Am J Hum Genet. 2006;78(6):999-1010.

van den Elzen ME, Twigg SR, Goos JA, Hoogeboom AJ, van den Ouweland AM, Wilkie AO, et al. Phenotypes of craniofrontonasal syndrome in patients with a pathogenic mutation in EFNB1. Eur J Hum Genet. 2014;22(8):9951001 .
Wallis D, Lacbawan F, Jain M, Der Kaloustian VM, Steiner CE, Moeschler JB, et al. Additional EFNB1 mutations in craniofrontonasal syndrome. Am J Med Genet A. 2008; 146A(15):2008-12.

Wieacker P, Horvath J. Craniofrontonasal Syndrome and EFNB1 Mutations. 3rd ed. In: Erickson RP, Wynshaw-Boris AJ, editors. Epstein's Inborn Errors of Development: The Molecular Basis of Clinical Disorders of Morphogenesis. Oxford University Press; 2016. p. 651-5.

Wieland I, Jakubiczka S, Muschke P, Cohen M, Thiele H, Gerlach KL, et al. Mutations of the ephrin-B1 gene cause craniofrontonasal syndrome. Am J Hum Genet. 2004;74(6):120915.

Wieland I, Reardon W, Jakubiczka S, Franco B, Kress W, Vincent-Delorme C, et al. Twentysix novel EFNB1 mutations in familial and sporadic craniofrontonasal syndrome (CFNS). Hum Mutat. 2005;26(2):113-8.

Wieland I, Weidner C, Ciccone R, Lapi E, McDonald-McGinn D, Kress W, et al. Contiguous gene deletions involving EFNB1, OPHN1, PJA1 and EDA in patients with craniofrontonasal syndrome. Clin Genet. 2007;72(6):50616.

Yousaf M, Ullah A, Azeem Z, Majeed AI, Memon MI, Ghous T, et al. Novel heterozygous sequence variant in the GLI1 underlies postaxial polydactyly. Congenit Anom (Kyoto). 2020;60(4):115-9. 